\title{
Round the clock work: the organisational issues
}

\author{
Bernard Glasson, Curtin University, West Australia, Chair \\ Dennis Bourque, Chevron Information Technology, USA
}

1

PANEL AIM

Global Inc., the 1997 IFIP WG 8.4 working conference, will operate "round-the-clock" and across the world. It will need a socio-technical infrastructure. Socio aspects include the organisation structure, the work flow, managing different time and different place work, synchronisation and version control, irregular working hours, multicultural teams, workplace management, and dealing with non-standard telecommunication interfaces and tool sets. If we call these the organisational aspects, then the aim of this panel is to surface the organisational issues associated with running Global Inc and to attempt to identify possible strategies for dealing with them. The "technical" aspects will be dealt with later in another panel.

\section{SOME EXAMPLES}

As an example let us consider work flow management and version control. In a distributed work environment it may be prudent to have one "region" (office or whatever) be responsible for a piece of work at any one time. Ownership may change as the work progresses (eg the problem is defined by one regional office, a solution to it is designed at another, the solution is built at a third and so on). Project ownership, albeit temporary, may bring with it rights and responsibilities. A right might be that of update. The other regions may read to project file, but the owner is the only one allowed to update it. And the responsibility might be to progress the work to a point where it can be handed on to the next region. At the hand-over of work from one region to the next the token or "baton" is passed to the next region which then assumes ownership rights and responsibilities. The "baton" may be one solution to an aspect of work flow management and an aspect of version control. The table below contains extracts from an earlier IFIP panel with a related theme which serve as further examples.

Dennis Bourque began proceedings with some short catalytic remarks. This was followed by a "brainstorm" session to surface the issues. Once the issues were raised and aired, the intention was to have open discussion in an attempt to identify possible solution strategies for dealing with the more significant of those issues. The intended outcome would be a list of issue statements and, hopefully, possible solution strategies which would be passed back to the Global Inc. design teams for their consideration. 
Table 1 Extracts from the panel discussion "Round the Clock Engineering" IFIP WG 8.1 Working Conference, Trondheim, Norway, August 1995

"More and more professionals are able to perform their daily work independent of their employer. In brief, physical distances are to a lesser and lesser degree serious obstacles for personal and professional communication....Engineering and construction of complex installations requires a number of different skills, and usually a large professional staff, with a corresponding need for extensive co-ordination of activities. Engineering projects have therefore traditionally been executed by one team, preferably located at one site where all the required skills were available...(but) it is no longer realistic to carry out (all) projects with remotely located teams. The cost, both in economic and social terms, are prohibitively high....The scenario is therefore that several groups of people, maybe alien to each other, and located in principle at an arbitrary distance from each other, often with disjoint working hours, shall cooperate over several months in order to produce one consistent set of specifications....The challenge is to combine distributed human skills in a synergetic manner....In a practical working environment, exchange of information is qualitatively different from data transfer." Helger Moen, Kvaerner Engineering, Norway

"Shift work was and still is unpopular with most people....Today around the clock working has a new meaning. We have an office in Houston, which is six hours behind (London) and an office in Yokohama which is 8 hours ahead....The opportunity now exists for performing work in three centres around the clock. In concept it should be possible to begin work on a design in London and then pass the effort around the world for as long as it takes to complete that design......In order for international around the clock working to be effective (we need to solve) a number of practical problems, some connected with the computer and others connected with ideas...the problems with communications of electronic documents are soluble, although transfer does take a considerable amount of time and must be carefully planned for. The problem that is less easy to solve is the transfer of ideas....for this we took advantage of the overlap in office times and teleconferencing facilities so that individuals could discuss matters face to face across a conferencing facility....It seems that there is nothing standing in the way of successful round the world engineering providing the economic will is there." Ron T Duckling, MV Kellogg Limited, UK

"A project management and engineering system designed for world-wide execution of multidisciplinary projects may cover a wide range of applications, systems and various organisations....the "time/activity/person" definition of ownership and responsibility for data becomes critical....Distribution of work may not only occur along the organisation, schedule and capability axes, but also along the time axis." Jorgen Piene, Kvaerner a s, Norway

"The distribution of the engineering process over multiple locations imposes additional requirements over those normally experienced at a single site operation: - Consistent language/terminology; consistent working practices and methods, including coding and numbering standards; consistency of tools and in particular software tools; efficient, reliable and secure voice and data networks; single source data; and efficient design change/revision control"

Steve Chatterton, Kvaerner H\&G Offshore Ltd, UK

"Now that the Engineering business is moving (..towards..) sending information back and forth across the Data Highway (.it..) creates a whole new set of problems: - What information do we have to send and in what format? What information do we receive back and in what format? How do we manage the outgoing and incoming information streams?.... Our world has shrunk....and we better learn to speak the global language. We therefore....(must develop and fully adopt)....international standards for our information exchange". Hans Teigeler, Fluor Daniel 


\section{POSTSCRIPT - THE PANEL OUTCOMES}

The thought-provoking input from Dennis Borque led to some the lively discussion between the 26 participants in the panel session. The outcome of that discussion was captured as eighteen "issues". These issues were then ranked in order of importance. There was not time to address the question of possible "solution strategies". The eighteen issues in order of perceived importance are summarised below along with one or two illustrative clarifying comments.

$1 \quad$ How do we maintain some form of coherence rather than disjointed disintegrated entries?

"This is a problem in face-to-face groups. We exhort planning and project management but live on-the fly. Can we expect to behave better when separated by time and space? Or, will we just become better at flaming?"

"encourage people to join in and see benefit - gain from this, don't throw the opportunity away"

2 How to create "focus" amongst people on different locations?

"The problem with not being together in "ill structured" tasks is that there is no natural focusing circumstance such as being in the same room together. For the Global Inc. event, this introduces a risk of superficiality. Solution direction: reduce ill-structuredness (eg. using "formal" brainwriting facilities or setting specific tasks such as playing a role in a game). Any other ideas?"

"Focus does not need to be just about location. What about drawing across several organisations? If a project team, working across time/space, is comprised of members from several organisations (or even several departments in one organisation), where is the loyalty when a paradox occurs between what is good for the project team, the person, and the organisation(s)? Time and space dislocation make this problem MORE critical than it is at this time."

3 How do you ensure everyone knows what they are supposed to be doing?

"This comment relates to the mere mechanics of the work that has to be done. Roles and responsibilities need to be clearly defined. When you are working across cultures, time zones, etc., co-ordination becomes difficult. Also when you have multiple centres of "control," coordination is difficult. So there needs to be some process or coordinating mechanism in place for ensuring that work is done by the correct people at the correct time."

4 How do we create and subsequently manage the archives/results/"memory" of the conference?

"Everyone who attends will have a different interpretation of what took place. How do we decide on what is the "official" record?..... Will all of the conference materials be available to anyone who wants to look at them, or will some of them have restricted access?.... Who does the "memory" belong to?" 
5 Should we (or do we want to) allow access to the conference from other than the 3 main sites? (e.g. voice/video/data)

"do we want to allow access to this conference to people at locations other than the three main sites - for example can I join the conference even if I am located at my home university?"

"I have a different (more nervous) point of view. This will be a first attempt for this community. We must ensure that what we tackle is achievable (eg organisers are volunteers, there is no obvious funding "pot of gold", must use the available technology etc. )"

6 How do we ensure that agreed tasks are done on time and what mechanisms do we need to ensure accountability?

"I think this comes under the same general topic as \#3 above"

7 The value of conferences is the networking

"Whole new cultural dimensions are emerging as a result of interactions based solely on electronic contact. Is this necessarily a bad thing? How can we effectively leverage this new culture?"

"I find many of the issues I "want" to talk about regarding global companies inappropriate for a one-shot conference, where almost no institutional relationships will persevere"

8 "personal presence": the need to interact directly with your co-workers for subtle communication and simulations

"If workers around the world are limited to only computer-mediated contact, will coordination, communication of goals, strategies and other subtle messages suffer?"

$9 \quad$ How do I foster informal communication when there is no "down time?"

"I value informal communication. Through this I can evaluate my contributions, my value, and my understanding of the work we, as a team, are enacting. Research and practice on how can I do this across time and space is mixed. How will I work across time and space when (1) there is no "time off" and (2) the work is critical to me, my team, and our organisation(s)? That is, when someone is always going, and things are constantly being added, how can I chat? For example, if round-the-clock work is a relay team, passing a "baton," then when did we practice? When do we finish the relay and debrief? How do we share?"

I like to meet people

"One of the greatest benefits for me regarding coming to a conference is meeting the people I have electronically spoken with - it helps build bonds and get to know what they are like better"

11 How do we establish and maintain sufficient trust among strangers?

"A better formulation of the original question might be (at least as far as generic work settings are concerned): How can we design and use technology to establish and reinforce collaborative relationships between people distributed in space and time, i.e. transform strangers to collaborators?" 
12 How do we overcome technical skill deficiencies? Proposal assumes a fairly high level of technical competence.

"Sounds like a good research topic right there :-)."

13 Nature of the work being done

"might itself raise challenges... might involve group discussion at times, information transmission, information exchange, etc..."

14 Work discipline

"If people are working at different places and times, it sometimes is very tempting to postpone activities you have to do for the 'group goal'. How do you encourage the participants' discipline with respect to group work?"

15 How can we differentiate proprietary and public knowledge?

"we are talking about issues to do with ideas, experience, concepts, frameworks"

"Everything you submit to a conference ...becomes public knowledge"

"But I might wish to archive some of the informal communications that occur at a conference and not have them available to everyone"

How do individuals receive credit for the work?

"When individuals work in teams they do not always contribute the same. In fact, one individual may contribute the fundamental "concept or thing" that makes the whole team activity work. Should this individual get credit for his/her input or should we care?"

17 How will the global connectivity be done (e.g., Internet, etc.\}?

"A technical issue!?"

18 Will Global Inc. lead to higher or lower quality "papers"?

"If the mode of work encourages folk to submit the number of submissions will go up making greater competition for fewer slots therefore (probably) the selected submissions will be of higher quality - conversely if it discourages submissions the "quality" might go down)"

"I think the quality of papers is one of the few things that won't be different for Global Inc. I mean, you're not going to sacrifice your current refereeing standards because we're getting global?? I think you should be careful to bring it to people's attention that paper quality is still one of the key factors that makes a conference a good conference." 